\title{
MR Imaging Assessment of Tumor Perfusion and 3D Segmented Volume at Baseline, during Treatment, and at Tumor Progression in Children with Newly Diagnosed Diffuse Intrinsic Pontine Glioma
}

\author{
J. Sedlacik, A. Winchell, M. Kocak, R.B. Loeffler, A. Broniscer, and C.M. Hillenbrand
}

\begin{abstract}
BACKGROUND AND PURPOSE: DIPG is among the most devastating brain tumors in children, necessitating the development of novel treatment strategies and advanced imaging markers such as perfusion to adequately monitor clinical trials. This study investigated tumor perfusion and 3D segmented tumor volume as predictive markers for outcome in children with newly diagnosed DIPG.
\end{abstract}

METHODS: Imaging data were assessed at baseline, during, and after RT, and every other month thereafter until tumor progression for 35 patients (ages 2-16 years) with newly diagnosed DIPG enrolled in the phase I clinical study, NCT00472017. Patients were treated with conformal RT and vandetanib, a vascular endothelial growth factor receptor 2 inhibitor.

RESULTS: Tumor perfusion increased and tumor volume decreased during combined RT and vandetanib therapy. These changes slowly diminished in follow-up scans until tumor progression. However, increased tumor perfusion and decreased tumor volume during combined therapy were associated with longer PFS. Apart from a longer OS for patients who showed elevated tumor perfusion after RT, there was no association for tumor volume and other perfusion variables with OS.

CONCLUSIONS: Our results suggest that tumor perfusion may be a useful predictive marker for the assessment of treatment response and tumor progression in children with DIPG treated with both RT and vandetanib. The assessment of tumor perfusion yields valuable information about tumor microvascular status and its response to therapy, which may help better understand the biology of DIPGs and monitor novel treatment strategies in future clinical trials.

ABBREVIATIONS: DIPG = diffuse intrinsic pontine glioma; $\mathrm{DSC}=$ dynamic susceptibility contrast; et $\mathrm{CO}_{2}=$ end-tidal carbon dioxide; $\mathrm{OS}=$ overall survival; $\mathrm{PFS}=$ progression-free survival; $\mathrm{RT}=$ radiation therapy

hildren with DIPG continue to have a very poor prognosis, with a median survival rate of less than 1 year. ${ }^{1,2}$ Standard therapy consists of conventionally fractionated RT, which only

Received August 7, 2012; accepted after revision October 17.

From the Departments of Radiological Sciences (J.S., A.W., R.B.L., C.M.H.), Biostatistics (M.K.), and Oncology (A.B.), St. Jude Children's Research Hospital, Memphis, Tennessee; and Department of Preventive Medicine (M.K.), University of Tennessee Health Science Center, Memphis, Tennessee.

Supported by the US National Institutes of Health Cancer Center Support (CORE) Grant No. P30 CA21765, AstraZeneca, and the American Lebanese Syrian Associated Charities (ALSAC), St. Jude Children's Research Hospital.

J.S. and A.W. contributed equally to this study.

Previously presented in part at: Annual Meeting of the American Society of Neuroradiology, May 17, 2010; Boston, Massachusetts; and Annual Meeting of the Inter national Society of Magnetic Resonance in Medicine, May 10, 2011; Montréal, Québec, Canada.

Please address correspondence to Claudia Hillenbrand, St. Jude Children's Research Hospital, 262 Danny Thomas Place, Mail Stop 220, Memphis, TN 38105; e-mail: claudia.hillenbrand@stjude.org

- Indicates open access to non-subscribers at www.ajnr.org

三 Indicates article with supplemental on-line tables.

http://dx.doi.org/10.3174/ajnr.A3421 temporarily improves the patients' clinical and neurologic status. The use of chemotherapy has shown no benefit in children with DIPG. The overall outcome of DIPG remains poor. ${ }^{2}$ In a phase I clinical study (NCT00472017) conducted at our institution, vandetanib (Caprelsa; AstraZeneca, Macclesfield, United Kingdom), a vascular endothelial growth factor receptor 2 and an epidermal growth factor receptor inhibitor, was given orally in conjunction with standard RT to children with newly diagnosed DIPG. ${ }^{3}$ To better understand the mechanisms of tumor growth, such as vascular proliferation, and response to therapy, we used advanced functional and anatomic MR imaging techniques to closely monitor tumor response and progression during this study.

An earlier imaging study of children with DIPG found no prognostic significance of conventional MR imaging assessment but suggested that quantitative parameters of advanced MR imaging techniques may serve as surrogate markers for therapy response and prognosis. ${ }^{4}$ Therefore, the objective of our prospective imaging study was to evaluate tumor perfusion, tumor blood volume, and high-resolution 3D segmented tumor volume as potential predictive markers for treatment response and tumor progression in children with newly diagnosed DIPG. 


\section{MATERIALS AND METHODS}

\section{Phase I Clinical Study}

The imaging study was conducted between June 2007 and August 2010 at our institution. ${ }^{3}$ A total of 35 patients (15 boys, 20 girls; median age, 6 years; age range, $2-16$ years) with newly diagnosed DIPG were enrolled. The maximal tolerated dose of vandetanib was determined by escalating dosage levels of $50(n=3), 65(n=$ $3), 85(n=3), 110(n=16)$, and $145(n=10) \mathrm{mg} / \mathrm{m}^{2}$ per day. Vandetanib was administered orally once daily and was continued for up to 2 years. Conformal RT was given in 1.8-Gy fractions 5 days a week for 6 weeks, for a total dose of 54 Gy to the planned target volume. Treatment with vandetanib and RT started on the same day. Corticosteroids were administered as necessary on an individual basis to control symptoms related to edema and mass effect, with the goal of discontinuing use when clinically applicable. The outcome of patients enrolled in the phase I study remained poor, with a 1 -year OS of $37.5 \% \pm 10.5 \%$ and a 2 -year OS of $21.4 \% \pm 11 \%$. Only 2 patients remain alive, with 1 patient free of progression 58 months after diagnosis. ${ }^{3}$

The institutional review board approved this protocol before initial patient enrollment, and continuing approval was maintained throughout the study. Written informed consent for participation was obtained from the parents or legal guardians of the patients, and assents were obtained when appropriate.

\section{MR Imaging}

MR imaging was scheduled at baseline (within 2 weeks before initiation of RT and vandetanib), $7 \pm 3$ days after the start of RT, and at the middle (week 3) and end of RT (week 6). Follow-up MR imaging examinations were assessed every other month after RT until the patient was taken off the study. Most patients were sedated during MR imaging. To account for the effect of sedation, ${ }^{5}$ diastolic blood pressure and etCO $\mathrm{CO}_{2}$ levels were recorded during MR imaging perfusion scans. MR examinations were performed mostly on a 3T Trio MR imaging scanner (Siemens, Erlangen, Germany). Because of relocation of the 3T MR imaging scanner during the study period, 3 patients mainly underwent MR examinations on a $1.5 \mathrm{~T}$ Avanto MR imaging scanner (Siemens) because their baseline examination was performed at 1.5T. Eight patients received 1 or 2 midtime course examinations on the $1.5 \mathrm{~T}$ scanner for the same reason. For longitudinal data uniformity, the 1.5T examinations were later removed from the data analysis. At each imaging time point, tumor perfusion was measured by use of both ASL and DSC-PWI. Tumor blood volume was also assessed by DSC-PWI. Tumor volume was evaluated on a T2-weighted high-resolution whole-brain $3 \mathrm{D}$ dataset acquired by a T2weighted 3D turbo spin-echo sequence with variable flip angles, ${ }^{6}$ with a TR of $3000 \mathrm{~ms}$ and a TE of $345 \mathrm{~ms}$. Automatic segmentation $^{7}$ of gray matter and WM was conducted on a T1-weighted high-resolution whole-brain 3D dataset acquired by magnetization-prepared rapid acquisition of gradient echo, ${ }^{8}$ with a TE of $2.44 \mathrm{~ms}$; TI, $900 \mathrm{~ms}$; and TR, $1920 \mathrm{~ms}$.

First, perfusion imaging was performed by use of a pulsed $\mathrm{ASL}^{9}$ sequence with an EPI readout consisting of 11 ascending sections with the following parameters: TR, $2280 \mathrm{~ms}$; TE, $23 \mathrm{~ms}$; matrix, $64 \times 64$; field of view, $210 \times 210 \mathrm{~mm}^{2}$; 5 -mm section thickness with 1-mm gap; and inversion times, $700 \mathrm{~ms}$ for TI1 and $1400 \mathrm{~ms}$ for TI2. A total of 50 label-control pairs and a spin attenuation map were collected and corrected for motion. ${ }^{10}$ Second, perfusion imaging was performed by use of DSC-PWI. Dynamic T2* -weighted images were acquired by EPI with 11 interleaved sections with the following parameters: TR, $1800 \mathrm{~ms}$; TE, $28 \mathrm{~ms}$; acquisition matrix, $64 \times 64$, interpolated by $k$-space zero-filling to $128 \times 128$; field of view, $210 \times 210 \mathrm{~mm}^{2}$; and $5-\mathrm{mm}$ section thickness with a $1-\mathrm{mm}$ gap. The EPI readout was repeated 90 times to image the first pass of the contrast agent bolus. DSC-PWI sections were positioned to anatomically coincide with ASL imaging sections. Baseline images were acquired for approximately 30 seconds, followed by a rapid intravenous bolus injection of $1-\mathrm{mL} / \mathrm{kg}$ gadopentetate dimeglumine (Magnevist, Berlex; Bayer HealthCare Pharmaceuticals, Wayne, New Jersey) with a power injector at a rate of $0.8-1.0 \mathrm{~mL} / \mathrm{s}$ (depending on the peripheral venous catheter size), followed immediately by a $20-\mathrm{mL}$ saline flush at the same rate. Approximately 5 minutes before the DSCPWI measurement, the extravascular space of the tumor was presaturated with the same $1-\mathrm{mL} / \mathrm{kg}$ gadopentetate dimeglumine dose to minimize potential compromising effects of a rapidly extravasating contrast agent. ${ }^{11}$

\section{Image Postprocessing}

ASL images were bicubically interpolated to a matrix size of $128 \times$ 128 to match the DSC-PWI images, and absolute ASL-CBF maps were calculated according to a previously reported method and were adjusted for intersection acquisition delays. ${ }^{12}$ The first-pass DSC-PWI data were processed by use of the PWI Task Card for SyngoMR (Athinoula A. Martinos Center, Massachusetts General Hospital, Boston, Massachusetts) by selection of a global arterial input from the middle cerebral arteries and calculation of perfusion and blood volume maps. ${ }^{13,14}$ Ratios of CBF and CBV were calculated between the median values of the 3D segmented tumor region of interest and the normal-appearing WM region of interest resulting from automated tissue segmentation. The mean ASL and DSC-PWI images, T1WI and T2WI, were coregistered by use of FLIRT ${ }^{15}$ (FMRIB Software Library; www.fmrib.ox.ac.uk/fsl). The tumor, defined as T2 hyperintense, was then manually segmented from the 3D high-resolution T2-weighted image. Applying the tumor region of interest as an exclusion mask, we automatically segmented the T1-weighted images by using $\mathrm{FAST}^{7}$ (FMRIB Software Library), generating 3D regions of interest of gray matter, WM, and CSF. We then transformed the tumor, gray matter, and WM regions of interest to the spatial resolution of DSC-PWI and interpolated ASL data, thereby facilitating sizematched tumor and tissue region-of-interest evaluation.

\section{Statistical Analysis}

The change in tumor perfusion and $3 \mathrm{D}$ volume from baseline to post-RT was investigated with use of the Wilcoxon signed-rank test. The changes in tumor perfusion and $3 \mathrm{D}$ volume were analyzed by 2 separate mixed-effects models: one from baseline to the end of RT and another from the end of RT to the end of study. We investigated the association between tumor volume and perfusion in a cross-sectional manner at each scheduled MR time point by using Spearman rank correlation (baseline and weeks 1, 3, 6, 16, 24 , and $26+$ from the start of therapy).

The association of tumor perfusion and volume with PFS and OS were investigated by use of Cox proportional hazards models. PFS was defined as the time from the start of therapy to the date of tumor progression or death, and OS was defined as the time from the start of therapy to death. Patients who did not have an event for PFS or OS were censored at their last follow-up dates. For a given variable of interest, the Cox survival model included the baseline assessment and the change during RT as fixed covariates, and the change following RT as a time-dependent covariate. To increase the usefulness of the ASL method, which measured per- 


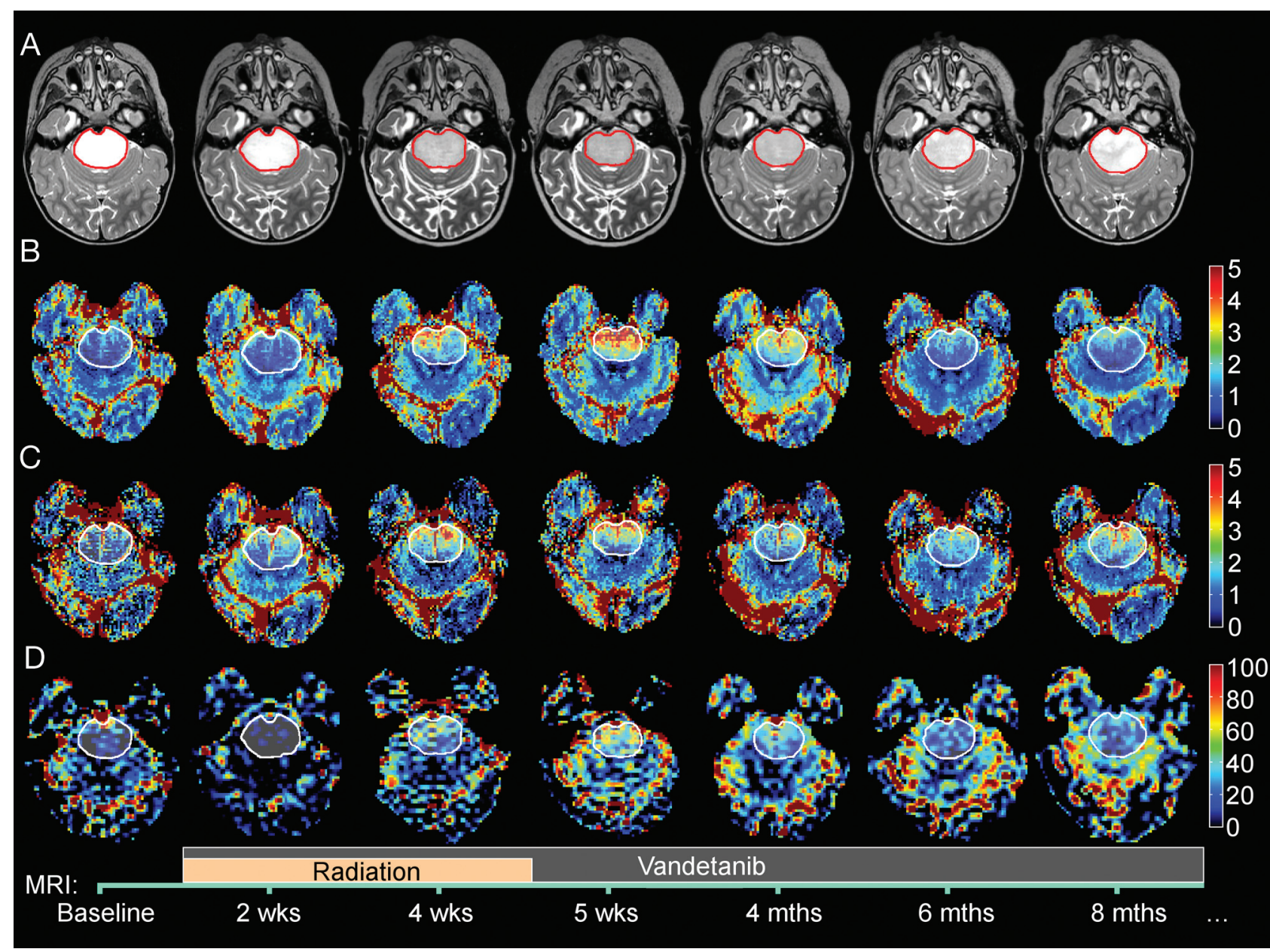

FIG 1. T2-weighted image with outlined tumor region of interest (row $A$ ) and corresponding maps of CBF (row $B$ ), CBV (row C), and ASL-CBF (row $D$, color bar of ASL-CBF is given in absolute units of milliliters per minute per 100-g tissue) of different time points during therapy. The course of therapy and time points of the images are depicted at the bottom. Changes of tumor tissue appearing in T2-weighted images and changes in tumor perfusion can be seen.

fusion values close to its noise levels in the image, we categorized tumor ASL-CBF values into 5 groups: group 1 (below sensitivity: ASL-CBF $<10 \mathrm{~mL} / \mathrm{min} / 100 \mathrm{~g}$ ), group 2 (comparable with WM: ASL-CBF 10 to $<30 \mathrm{~mL} / \mathrm{min} / 100 \mathrm{~g}$ ), group 3 (between gray matter and WM: ASL-CBF 30 to $<50 \mathrm{~mL} / \mathrm{min} / 100 \mathrm{~g}$ ), group 4 (comparable with gray matter: ASL-CBF 50 to $<70 \mathrm{~mL} / \mathrm{min} / 100 \mathrm{~g}$ ), and group 5 (above gray matter: ASL-CBF $>70 \mathrm{~mL} / \mathrm{min} / 100 \mathrm{~g}$ ).

To account for the effect of sedation, ${ }^{5}$ we studied the associations of tumor perfusion with physiologic measures of etCO $\mathrm{CO}_{2}$ and diastolic blood pressure in a cross-sectional fashion by using Spearman rank correlation coefficients and in a longitudinal fashion by using mixed-effects models.

Given the exploratory nature of these studies, the $P$ values were not adjusted for multiple testing, and the findings must be considered in a hypothesis-generating context.

\section{RESULTS}

Of the 35 patients enrolled in the study, 2 patients had shunts, which caused image artifacts and compromised quantitative MR imaging perfusion measurements. These patients were excluded from the analysis. Overall, $205 \mathrm{MR}$ examinations from 33 patients were processed, of which 28 scans were acquired at $1.5 \mathrm{~T}$ and were therefore removed from the final analysis to maintain standardization of image data acquisition. Three patients who had mainly
1.5T examinations were removed completely. Of the remaining 30 patients, 1753 T MR examinations were analyzed. There were 3 patients with 8 examinations each, 8 patients with 7 examinations, 10 patients with 6 examinations, 4 patients with 5 examinations, 2 patients with 4 examinations, 1 patient with 3 examinations, and 2 patients with 2 examinations. Of the remaining 30 patients, 29 had a baseline scan and at least 1 on-treatment scan, and 22 patients had a baseline scan and an immediate post-RT scan; thus, pre-RT vs post-RT comparisons were only possible for this cohort of 22 patients.

\section{MR Imaging}

Qualitative image assessment showed a bright tumor signal in T2-weighted images at baseline, which decreased during the end of RT, accompanied by a decrease in 3D tumor volume (Fig 1, row A). After RT, the T2-weighted signal of the tumor became hyperintense again, and the tumor volume also increased. The analyzed tumor region of interest outlined in red on the T2-weighted image was transformed to the perfusion maps outlined in white (Fig 1, rows $\mathrm{B}-\mathrm{D}$ ). Median changes in tumor $\mathrm{CBF}, \mathrm{CBV}$, and ASL-CBF plotted as time progressed showed a distinct elevated tumor perfusion immediately after RT (Fig 2, box plots). Wilcoxon signedrank tests revealed that these increases in tumor ASL-CBF $(P=$ $.006)$, $\mathrm{CBF}(P<.0001)$, and $\mathrm{CBV}(P<.0001)$ were statistically 
significant (On-line Table 1). On the contrary, there was a significant decrease $(P<.0001)$ in 3D tumor volume between baseline and the immediate post-RT scan (On-line Table 1).

To evaluate the changes of tumor parameters with time during treatment and follow-up, we constructed 2 separate mixed-effects models for all time points before the end of RT (baseline and during RT) and after RT. The combined average fit of these 2 models is illustrated in Fig 2 by the dashed red lines. Details of the models are presented in On-line Table 2. Our model suggested a quadratic behavior $(P<.0001)$ for tumor ASL-CBF during RT: it first decreased and then increased during RT, followed by a gradual linear decline after RT $(P=.0002)$. Tumor volume, on the contrary, showed an opposite trend: it had a nonlinear decline during RT $(P=.0001)$ and then a gradual nonlinear increase after RT $(P=.0002)$. Both CBF and CBV linearly increased during RT $(P=.0003$ and $P=.0001$, respectively), followed by a gradual linear decrease $(P=.0015$ and $P=.0035$, respectively) after RT. A cross-sectional Spearman rank correlation analysis between tumor perfusion and tumor volume showed no convincing overall association of tumor perfusion with tumor volume, except for a suggestive positive correlation between $\mathrm{CBF}$ and tumor volume at the end of RT (On-line Table 3).

\section{Survival Analysis}

Survival analysis on the basis of Cox proportional hazards models showed no significant association of vandetanib dose, age at diagnosis, or sex with PFS ( $P$ values $=.065, .10$, and .075 , respectively), or with OS ( $P$ values $=.125, .18$, and .11 , respectively). Cox model results for the association of categorized ASL-CBF, $\mathrm{CBF}, \mathrm{CBV}$, and tumor volume with PFS and OS are presented in On-line Table 4. These multivariable survival models suggest that patients with higher CBF at baseline have longer PFS $(P=.0061$ and $P=.011$ for categorized ASL-CBF and CBF, respectively), keeping the other covariates in the model fixed. Also, patients with higher increase in CBF during RT had more favorable PFS than those who had lower increase or decrease in CBF during RT $(P=.044$ and $P=.0057$ for categorized ASL-CBF and CBF, respectively), keeping the other covariates fixed. After RT, categorized ASL-CBF and CBF were both positively associated with a more favorable PFS ( $P=.0034$ and $P=.001$, respectively). Keeping the other covariates fixed, patients with a steeper decline in tumor perfusion had shorter PFS on average, whereas patients with slower decline or an incline in CBF had more favorable PFS on average.

In addition, larger tumor volumes at baseline were associated with more favorable PFS $(P=.013)$ than smaller tumor volumes, likely because larger tumors at baseline tended to have better RT response (Spearman rank correlation of 0.61 with $P<.001$, comparing pre-RT and immediately post-RT tumor volume measurements). Patients with smaller decline or increase in tumor volume during RT had less favorable PFS $(P=.019)$ than those with a greater decline in tumor volume. As expected, an increase in tumor volume after RT led to earlier progression overall $(P<$ .0001). The multivariable models did not indicate any statistical association of changes in the CBV of the tumor with PFS or OS.

None of the tumor volume or CBF measurements at baseline, during RT, or post-RT was significantly associated with OS. However, CBF indicated that patients with higher tumor perfusion at baseline had slightly more favorable OS $(P=.041)$ (On-line Table 4) than patients with lower tumor perfusion at baseline.

\section{Sedation Effects}

Cross-sectional analysis of the association between tumor perfusion and physiologic measures evaluated to account for the effect of sedation ${ }^{5}$ did not reveal a significant correlation, except for etCO $\mathrm{C}_{2}$ and $\mathrm{ASL}-\mathrm{CBF}$ at week $3(P=.017)$ (On-line Table 5). Longitudinal post-RT evaluations by the mixed-effects models showed some evidence that etCO $\mathrm{C}_{2}$ is associated with ASL-CBF $(P=.03)$. ASL-CBF changes pre-RT to post-RT were not associated with changes in etCO $\mathrm{O}_{2}$ during RT (Spearman rank correlation of 0.23 with $P=.32$ ).

\section{DISCUSSION}

Because the overall outcome of DIPGs remains poor, the development of novel treatment strategies and advanced imaging markers to adequately monitor clinical trials is of high importance. To better understand the mechanisms of tumor growth, such as vascular proliferation, and their response to therapy, we closely monitored tumor perfusion and tumor volume of patients with newly diagnosed DIPGs treated with combined conventionally fractionated conformal radiation and antiangiogenic therapy.

We found an increase in median tumor perfusion and blood volume and a decrease in tumor volume at the end of RT. After RT, the elevated tumor perfusion and blood volume steadily decreased with time, with tumor volume increasing concurrently. The observed quadratic time dependency of ASL-CBF during RT, as opposed to the linear time dependency of DSC-CBF, may be caused by the different measures of absolute quantitative tumor perfusion by ASL and the relative quantitative ratio between tumor and WM perfusion by DSC. As expected, there was a significant association of decreased tumor volume during RT and maintenance of low tumor volumes thereafter with longer PFS, which demonstrates that tumor volume is still an important determinant in the evaluation of treatment response. Also, in our study progression was based on tumor size, ${ }^{16}$ which was assessed during the regular reading of conventional axial and sagittal 2D MR imaging by pediatric neuroradiologists, if the product of the maximal perpendicular diameters of the tumor increased by more than $25 \%$ compared with baseline. The association of larger tumor volumes at baseline with longer PFS in our study agrees with findings of the Pediatric Brain Tumor Consortium , ${ }^{17}$ which may be because larger tumors at baseline showed a higher decrease in tumor volume at the end of RT, indicating a better response to therapy. Poussaint et $\mathrm{al}^{17}$ suggested that these larger tumors at baseline may represent less aggressive neoplasm because they were allowed to slowly grow to a larger size before clinical detection. The absence of association between 3D tumor volume and OS is in agreement with previous studies on DIPG. ${ }^{4}$

We found no direct association between tumor perfusion and 3D tumor volume; therefore, the known interdependency among tumor size, tumor interstitial pressure, and tumor perfusion may not explain our observations. ${ }^{18,19}$ However, the confined localization of the tumor may confound the correlation analysis between tumor volume and perfusion. The qualitatively observed decrease in the T2-weighted tumor signal (Fig 1) may suggest that diffusion imaging would be useful in elucidating if changes in tumor perfusion are secondary effects of changes of edema and, therefore, interstitial pressure in the tumor. ${ }^{20}$

Patients who had higher tumor perfusion had longer PFS than those who had lower tumor perfusion. This association was also found for OS and CBF at baseline. These results suggest that tumor perfusion may be a useful prognostic factor for progression and outcome in DIPG treated with RT and vandetanib. Our findings are 

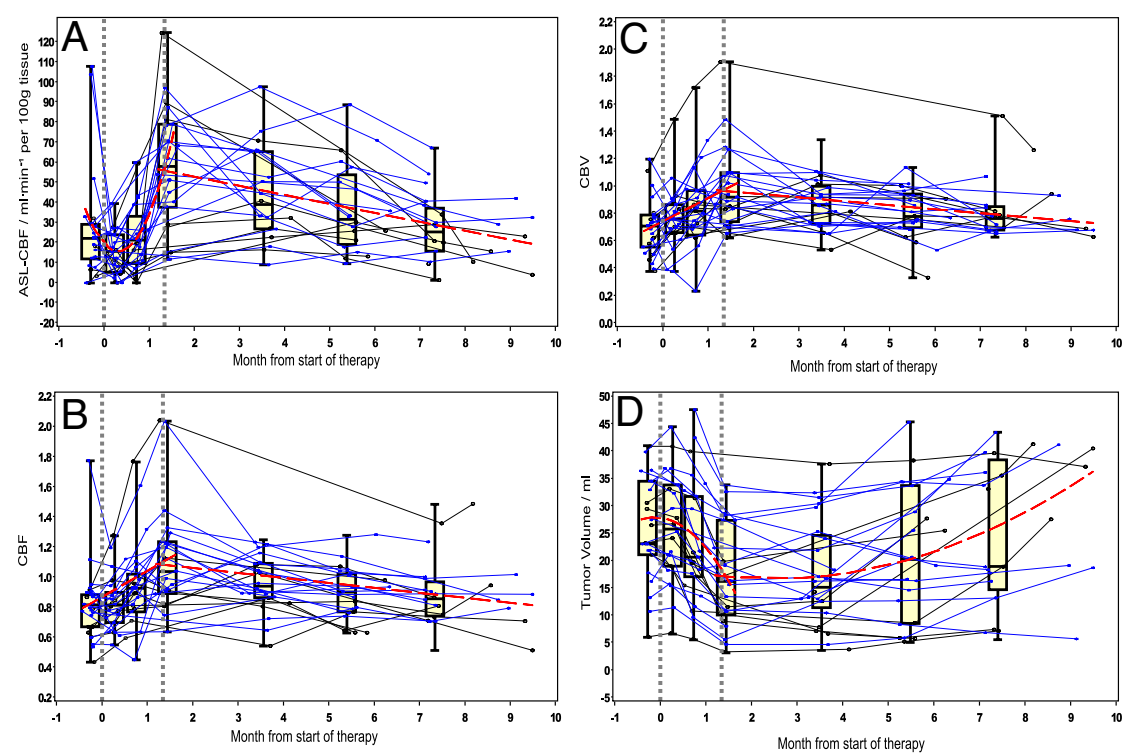

FIG 2. Time course of $A S L-C B F(A), C B F(B), C B V(C)$, and tumor volume $(D)$ during and after RT. Beginning and end of RT are marked by dotted straight gray lines at time points 0 and 1.4 months. Red bold dashed curves indicate the time trend of the most statistically significant linear or quadratic model. Solid lines denote individual time course of each patient and patients who used steroids after RT are marked in blue. Box plots were overlaid to highlight the distribution of values at each time point.

consistent with those of a recent study showing increased survival duration in patients with glioblastoma who respond to antiangiogenic therapy with elevated blood perfusion. ${ }^{21}$ However, because we did not have a control group in our phase I study and the natural perfusion history of this tumor is not known, we are not able to determine whether this observation of increased perfusion with longer PFS and OS was a result of the antiangiogenic therapy, ${ }^{20} \mathrm{RT}$ alone, or the combination of these 2 treatments. Furthermore, these results could also have been found to be very different for patients receiving RT only or for untreated patient cohorts. Nevertheless, we are very confident that our findings are a true effect of therapy because increased tumor perfusion coincides with the onset of treatment and the decrease in tumor volume.

The median CBV ratio of the whole tumor region of interest was not significantly associated with PFS or OS. The measured $\mathrm{CBV}$ ratio was less than or approximately 1 , in contrast to a CBV of more than 1.75 observed in high-grade gliomas, which was a prognostic factor of poor outcome. ${ }^{22}$ A recent study recommending the use of DSC-PWI imaging in DIPG showed that increased blood volume at baseline or on follow-up examinations was associated with reduced survival duration. ${ }^{23}$ However, in our study blood volume was used as a continuous variable, but Hipp et $\mathrm{al}^{23}$ evaluated blood volume as a binary variable with increase or decrease in blood volume as the 2 values. In another study, small distinct areas of focal anaplasia within DIPG were identified and were characterized by a higher CBV than that of the rest of the tumor. ${ }^{24}$ Therefore, data evaluation in the future should investigate the heterogeneity ${ }^{25}$ of $\mathrm{CBV}$ values inside the whole tumor region of interest or in specific subregions of the tumor to test for its potential prognostic value.

To account for the confounding effect of etCO $\mathrm{CO}_{2}$ on cerebral perfusion in sedated, spontaneously breathing patients, ${ }^{5}$ etCO $_{2}$ levels were recorded during perfusion imaging. Cross-sectional analysis revealed a positive association of etCO $\mathrm{CO}_{2}$ and ASL-CBF for the first 3 time points (baseline, week 1, and week 3), and a lon- gitudinal analysis after RT also found a significant association between etCO and ASL-CBF. However, this association was not observed in relative CBF (DSCPWI), which suggests that the vasodilative effect of $\mathrm{CO}_{2}$ may have a similar effect on both DIPG and WM perfusion. Thus, relative perfusion measures are not as sensitive with respect to the systemic effect of $\mathrm{CO}_{2}$. Because of the similarities in the longitudinal trends of tumor perfusion measured by both ASL and DSC-PWI, we believe that our findings for ASL-CBF with PFS and OS are still meaningful and are not compromised by etCO $\mathrm{O}_{2}$. Nevertheless, future studies should try to maintain a fixed et $\mathrm{CO}_{2}$ level during perfusion measurement to avoid this potentially confounding factor.

A potential limitation of our study was the use of steroids, which were given on an individual basis and were not recorded in detail during the study. In retrospect, we could only identify patients who used steroids at 1 point after RT; these patients are indicated with asterisks and blue lines in Fig 2. Therefore, use and dosage of steroids as a possible confounding factor could not be assessed in our analysis. Future studies should investigate the use of steroids in the evaluation of tumor characteristics. Furthermore, our EPIASL technique had low signal-to-noise ratio and was prone to artifacts from field inhomogeneities in the area of the brain stem. Therefore, the use of more advanced and robust ASL techniques such as $3 \mathrm{D}-\mathrm{GRASE}-\mathrm{ASL}^{26}$ with background suppression or

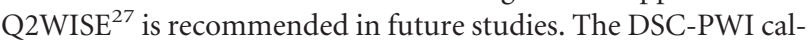
culation used a global arterial input that was selected from the MCA far away from the location of the tumor. Newer techniques that use a local arterial input that closely resembles a pontine arterial input function could improve the reliability of CBF and CBV measurements. ${ }^{28}$ To increase the prognostic usefulness of tumor perfusion, both diffusion and perfusion measurement should be made in conjunction to differentiate the possible effects of tumor edema. Furthermore, because of the long period in which the study was performed (ie, relocation of MR scanners), data standardization and consistency were of particular interest and may have been potential limitations of our study.

Because of the relocation of the 3T scanner during our study period, some examinations were performed at $1.5 \mathrm{~T}$ and were removed from the statistical analysis to eliminate any potential confounding factors arising from different field strength. This exclusion criterion also removed 3 patients who had mainly $1.5 \mathrm{~T}$ examinations. Eight other patients had a mixture of $1.5 \mathrm{~T}$ and $3 \mathrm{~T}$ examinations. We chose to keep the $3 \mathrm{~T}$ examinations of those 8 patients in the statistical analysis because the examinations are independent of each other, and all 8 patients had 3T examinations at baseline. The removal of these 8 patients would have led to an overall sample size that would have been too limiting for our longitudinal and survival models.

\section{CONCLUSIONS}

Our results suggest that tumor perfusion may be a useful predictive marker for the assessment of treatment response and tumor 
progression in children with DIPG treated with RT and vandetanib. The assessment of tumor perfusion yielded valuable information about the tumor's microvascular status and its response to therapy, which may help to better understand the biology of DIPGs and monitor novel treatment strategies in the future.

\section{ACKNOWLEDGMENTS}

We thank Dr. Arzu Onar-Thomas, Department of Biostatistics, St. Jude, for critical review of the manuscript and Dr. Vani Shanker, Department of Scientific Editing, for editorial review. We would also like to thank Dr. Thomas Benner and Massachusetts General Hospital, Boston, for providing us with the PWI task card.

Disclosures: Alberto Broniscer—RELATED: Grant: AstraZeneca, ${ }^{\star}$ Comments: AstraZeneca provided the study agent and also some financial support for the respective clinical trial; Provision of Writing Assistance, Medicines, Equipment, or Administrative Support: AstraZeneca (see above).* *Money paid to institution.

\section{REFERENCES}

1. Broniscer A, Gajjar A. Supratentorial high-grade astrocytoma and diffuse brainstem glioma: two challenges for the pediatric oncologist. Oncologist 2004;9:197-206

2. Hargrave D, Bartels U, Bouffet E. Diffuse brainstem glioma in children: critical review of clinical trials. Lancet Oncol 2006;7:241-48

3. Broniscer A, Baker JN, Tagen M, et al. Phase I study of vandetanib during and after radiotherapy in children with diffuse intrinsic pontine glioma. J Clin Oncol 2010;28:4762-68

4. Hargrave D, Chuang N, Bouffet E. Conventional MRI cannot predict survival in childhood diffuse intrinsic pontine glioma. J Neurooncol 2008;86:313-19

5. Sedlacik J, Lobel U, Kocak M, et al. Attenuation of cerebral venous contrast in susceptibility-weighted imaging of spontaneously breathing pediatric patients sedated with propofol. AJNR Am J Neuroradiol 2010;31:901-06

6. Mugler III JP, Wald LL, Brookeman JR. T2-weighted 3D spin echo train imaging of the brain at 3 Tesla: Reduced power deposition using low flip-angle refocusing RF pulses. In: Proceedings of the International Society for Magnetic Resonance Imaging; April 21-27, 2001; Glasgow, United Kingdom. Abstract 438

7. Zhang Y, Brady M, Smith S. Segmentation of brain MR images through a hidden Markov random field model and the expectationmaximization algorithm. IEEE Trans Med Imaging 2001;20:45-57

8. Mugler JP 3rd, Brookeman JR. Three-dimensional magnetizationprepared rapid gradient-echo imaging (3D MP RAGE). Magn Reson Med 1990;15:152-57

9. Luh WM, Wong EC, Bandettini PA, et al. QUIPS S II with thin-slice TI1 periodic saturation: a method for improving accuracy of quantitative perfusion imaging using pulsed arterial spin labeling. Magn Reson Med 1999;41:1246-54

10. Thesen S, Heid O, Mueller E, et al. Prospective acquisition correction for head motion with image-based tracking for real-time fMRI. Magn Reson Med 2000;44:457-65

11. Lüdemann L, Warmuth C, Plotkin M, et al. Brain tumor perfusion: comparison of dynamic contrast enhanced magnetic resonance imaging using $\mathrm{T} 1, \mathrm{~T} 2$, and $\mathrm{T} 2{ }^{\star}$ contrast, pulsed arterial spin labeling, and H2(15)O positron emission tomography. Eur J Radiol 2009;70: 465-74

12. Wang J, Alsop DC, Li L, et al. Comparison of quantitative perfusion imaging using arterial spin labeling at 1.5 and 4.0 Tesla. Magn Reson Med 2002;48:242-54

13. Ostergaard L, Sorensen AG, Kwong KK, et al. High resolution measurement of cerebral blood flow using intravascular tracer bolus passages. Part II: experimental comparison and preliminary results. Magn Reson Med 1996;36:726-36

14. Ostergaard L, Weisskoff RM, Chesler DA, et al. High resolution measurement of cerebral blood flow using intravascular tracer bolus passages. Part I: mathematical approach and statistical analysis. Magn Reson Med 1996;36:715-25

15. Jenkinson M, Bannister P, Brady M, et al. Improved optimization for the robust and accurate linear registration and motion correction of brain images. Neuroimage 2002;17:825-41

16. Macdonald DR, Cascino TL, Schold SC Jr, et al. Response criteria for phase II studies of supratentorial malignant glioma. J Clin Oncol 1990;8:1277-80

17. Poussaint TY, Kocak M, Vajapeyam S, et al. MRI as a central component of clinical trials analysis in brainstem glioma: a report from the Pediatric Brain Tumor Consortium (PBTC). Neuro Oncol 2011; 13:417-27

18. Gutmann R, Leunig M, Feyh J, et al. Interstitial hypertension in head and neck tumors in patients: correlation with tumor size. Cancer Res 1992;52:1993-95

19. Znati CA, Rosenstein M, Boucher $Y$, et al. Effect of radiation on interstitial fluid pressure and oxygenation in a human tumor xenograft. Cancer Res 1996;56:964-68

20. Jain RK, Tong RT, Munn LL. Effect of vascular normalization by antiangiogenic therapy on interstitial hypertension, peritumor edema, and lymphatic metastasis: insights from a mathematical model. Cancer Res 2007;67:2729-35

21. Sorensen AG, Emblem KE, Polaskova P, et al. Increased survival of glioblastoma patients who respond to antiangiogenic therapy with elevated blood perfusion. Cancer Res 2012;72:402-07

22. Law M, Young RJ, Babb JS, et al. Gliomas: predicting time to progression or survival with cerebral blood volume measurements at dynamic susceptibility-weighted contrast-enhanced perfusion MR imaging. Radiology 2008;247:490-98

23. Hipp SJ, Steffen-Smith E, Hammoud D, et al. Predicting outcome of children with diffuse intrinsic pontine gliomas using multiparametric imaging. Neuro Oncol 2011;13:904-09

24. Löbel U, Sedlacik J, Reddick WE, et al. Quantitative diffusionweighted and dynamic susceptibility-weighted contrast-enhanced perfusion MR imaging analysis of T2 hypointense lesion components in pediatric diffuse intrinsic pontine glioma. AJNR Am J Neuroradiol 2011;32:315-22

25. Young R, Babb J, Law M, et al. Comparison of region-of-interest analysis with three different histogram analysis methods in the determination of perfusion metrics in patients with brain gliomas. J Magn Reson Imaging 2007;26:1053-63

26. Günther M, Oshio K, Feinberg DA. Single-shot 3D imaging techniques improve arterial spin labeling perfusion measurements. Magn Reson Med 2005;54:491-98

27. Song R, Loeffler RB, Hillenbrand CM. QUIPS S II with windowsliding saturation sequence (Q2WISE). Magn Reson Med 2012;67: 1127-32

28. Willats L, Christensen S, Ma HK, et al. Validating a local Arterial Input Function method for improved perfusion quantification in stroke. J Cereb Blood Flow Metab 2011;31:2189-98 